\title{
On the Stagnant Zone Upstream of an Obstacle within a Stably Stratified Flow in the Atmosphere
}

\author{
By Koji Kitabayashi \\ National Research Institute for Pollution and Resources, Yatabe, Ibaraki 305 \\ (Manuscript received 19 August, 1980, in revised form 3 March 1981)
}

\begin{abstract}
Stably stratified airflows over a hill or mountain are studied by a wind tunnel and also by a theoretical model with special forcus on the upstream stagnant zone. The wind tunnel experiment shows that a stagnant zone is formed upstream of an obstacle when the Froude number is less than some critical value. The critical Froude number is found to vary with the obstacle height. In the stagnant zone, the airflow is negligible or reverse and the thickness of the zone decreases gradually with the distance from the obstacle.
\end{abstract}

\section{Introduction}

When a stably stratified airflow goes over a mountain, well known lee waves develop downstream. Many theoretical studies have been reported for lee wave problems, but most of the models assumed that the air mass flows over the mountain and no blocking or stagnation of air flow occurs upstream. Sheppard (1956) first suggested that flow blockage may occur depending on the flow conditions by the balance of momentum and buoyancy forces.

A theoretical model of the upstream stagnant zone was first proposed by Kao (1965). He solved a Helmholz type equation which was derived by Yih (1958) for non-diffusive, nonviscous, linear density-gradient flow over an obstacle in a channel, assuming that the stagnant zone is a completely still flow region. In his theoretical model, the obstacle is produced by virtual sinks and vortices. Kao insisted that a stagnant zone is formed when an internal Froude number based on the channel height is less than $1 / \pi$. He did not discuss the dependency of the critical Froude number on the obstacle height. As suggested by Sheppard, the critical Froude number should be dependent on the obstacle height, i.e. under an equal stability condition, the flow blockage may be formed for higher obstacle rather than smaller one.

In observational or experimental studies of the upstream stagnant zone, only a wind tunnel and field study by the present author (1977) is available. He studied the flow stagnation upstream of a two-dimensional model hill within a ground level inversion and suggested that Froude number with respect to the obstacle height is a good controlling parameter.

The flow stagnation of a sink flow was studied by Hino and Onishi (1970) concerning the problem of selective withdrawal, and it was clarified that the critical Froude number is dependent upon the position of the sink. The critical value was shown to take the maximum when the sink is just at the middle level of the channel depth.

In this study, we simplify hills or mountains to a thin vertical barrier to exclude the direct influences of the hill shape and slope angle and study the airflow over it by a wind tunnel experiment and also by a theoretical model. The dependency of the critical Froude number on the barrier height and also changes of the flow properties due to the stability parameter are discussed.

\section{Experimental apparatus and procedures}

\subsection{The wind tunnel facility}

The present experiments have been done in a wind tunnel of National Research Institute for Pollution and Resources. The wind tunnel is an open circuit type with a test section of 5 meters long, 0.6 by 0.6 meters in cross section. A side 
(a)

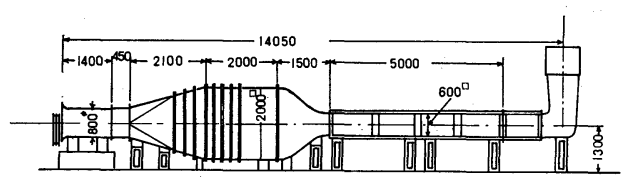

(b).

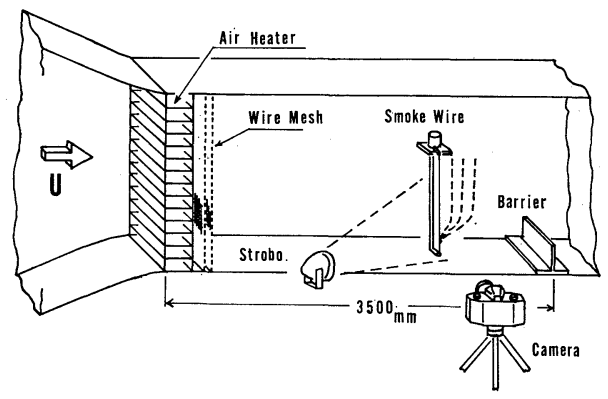

Fig. 1(a) Wind tunnel and dimensions.

(b) Experimental set up.

of the test section consists of hinged glass doors (Fig. 1). The disposition of the barrier and measuring system is also shown in Fig. 1.

The density stratification has been produced by an air heater system installed at the inlet of the test section. The system is composed of thirteen elements of plate heaters, each of them is set horizontally at $4 \mathrm{~cm}$ intervals. By changing the electric current supplied to each element, differential heating of airflow is done and temperature gradient is produced. By this system, the temperature of the airflow inside the wind tunnel becomes about $30^{\circ} \mathrm{K}$ higher than the outer air temperature at wind velocity of $0.5 \mathrm{~m} / \mathrm{s}$.

\subsection{The smoke wire system}

The flow velocity has been measured by a smoke wire system. It is a kind of Lagrangian method and may not be affected by temperature or density heterogeneity. This method is suitable for the measurements of non-turbulent low velocity flow. In this experiment, the smoke wire was covered by short pipes of $1 \mathrm{~mm}$ thick and $10 \mathrm{~mm}$ long, and the vertical component of the velocity is also detectable from the vertical transport of smoke pieces. The smoke pieces were photographed sequentially after the emission of them. For the lighting of the smoke, a strobo flash was used. The time duration of the strobo was set at 0.1 or 0.2 second depending on the flow velocity.

\subsection{The models and experimental procedure}

A thin vertical barrier was used as the obstacle. To clarify the effects of the barrier height, three barriers were made of plywood of $10 \mathrm{~mm}$ thick. They are 5, 11 and $15 \mathrm{~cm}$ high or equal to 0.08 , 0.18 and 0.25 in normalized height $b$ (the barrier height $h$ normalized by the depth of the test section of the wind tunnel $H$, i.e. $b=h / H$ ), respectively. The width of the model was equal to that of the test section, so that the two-dimensionality of the flow was established well. The barrier was set $3.5 \mathrm{~m}$ downstream of the inlet of the test section. The measurements of the velocity profiles were started after the temperature profile of the airflow reached steady state.

The obtained data were analyzed with respect to the internal Froude number $F_{r \pi}$, which is defined by

$$
F_{r_{\pi}}=\frac{U_{\infty} \pi}{N H},
$$

where $N$ is the well-known Brunt-Väisälä frequency defined at upstream infinity $x=-\infty$ and written by

$$
N^{2}=\frac{g}{T_{0}}\left(\frac{\partial T}{\partial z}\right)_{x=-\infty} .
$$

In equations (2-1) and (2-2), $U_{\infty}$ is the reference velocity, $g$ is the gravitational acceleration, $T$ and $T_{0}$ are local and reference temperature of the airflow and $z$ is the vertical coordinate. For the airflow in the atmosphere, $T$ and $T_{0}$ are replaced by the potential temperature.

\section{The experimental results}

\subsection{Airflow upstream of the barrier}

To clarify the effect of density stratification on the airflow over the barrier, velocity profiles in non-stratified condition were surveyed first. Fig. 2(a) and (b) are the smoke pictures taken at 10 and $40 \mathrm{~cm}(x / h=1.0$ and 4.0 respectively) upstream of the barrier of $b=0.18$. The flow velocity was $40 \mathrm{~cm} / \mathrm{s}$ and the time duration of the strobo flash was $0.1 \mathrm{sec}$. These pictures were taken by keeping the shutter of the camera open, generating the smoke and flashing the strobo several times serially in a given time duration.

The smoke picture at $x / h=1.0$ shows the influence of the barrier, i.e. the flow velocity is recognized to decrease in the lower layer. At $x / h=4.0$, the smoke pieces line up vertically. This means that the velocity is constant with height and the influence of the barrier is almost negligible at this position.

Fig. 3(a) and (b) are the smoke pictures for the barrier of $b=0.25$ in the stably stratified flow with $F_{r \pi}$ equal to 0.71 . The flow is from right to left in the pictures. These pictures were taken 


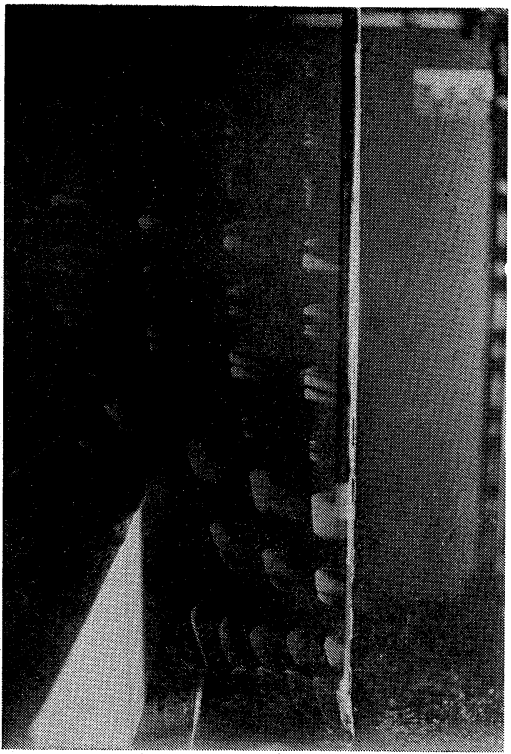

(a)

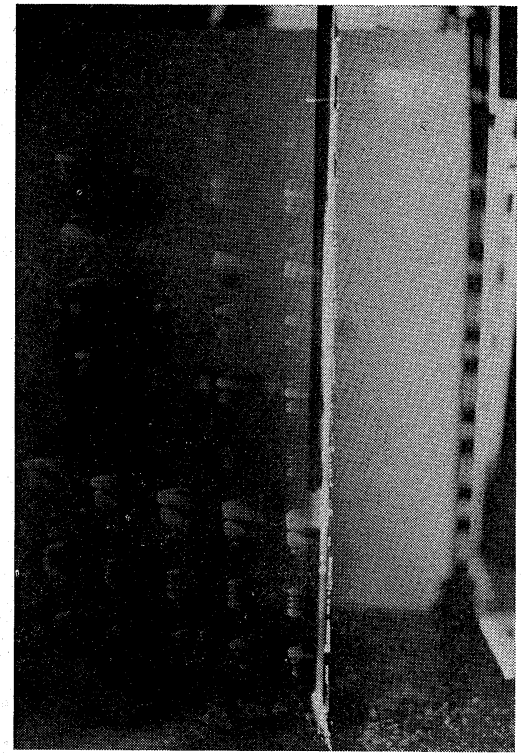

(b)

Fig. 2 Smoke pictures upstream of the barrier of $b=0.18$ for the non-stratified flow. The flow is from right to left. Time duration of strobo flash is $0.1 \mathrm{sec}$. (a) $x / h=1.0$; (b) $x / h=4.0$.

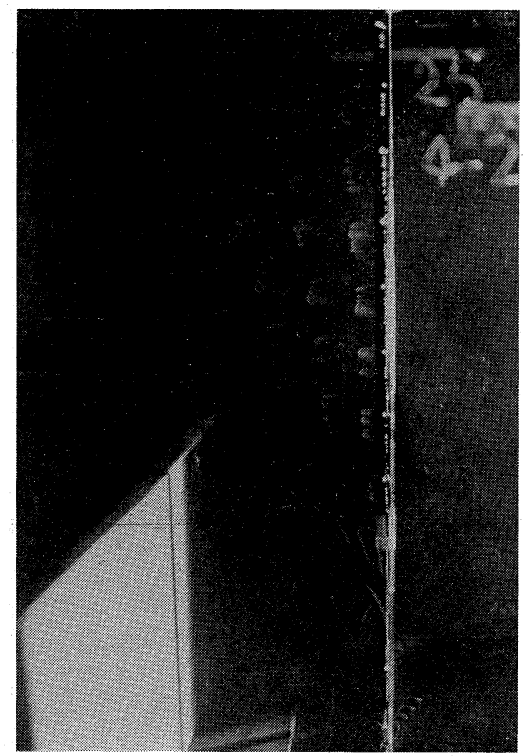

(a)

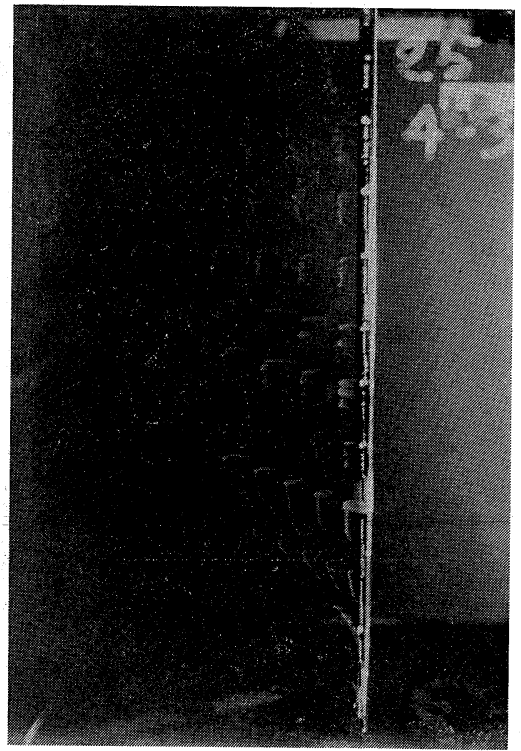

(b)

Fig. 3 Smoke pictures for stably stratified condition. $F r \pi=0.71, b=0.25$. (a) $x / h=0.67$; (b) $x / h=2.67$.

at $10 \mathrm{~cm}(x / h=0.67)$ and $40 \mathrm{~cm}(x / h=2.67)$ up- stream.

stream of the barrier respectively. The movement of the smoke pieces in the lower layer is negligible at $x / h=0.67$ and also at $x / h=2.67$ and a are shown in Fig. 4(a) and (b). The upstream stagnant zone is considered to be formed. Here, positions of the smoke wire in Fig. 4(a) and (b) we define the stagnant zone as the region where are $x^{\prime} / h=2.0$ and 4.0 , respectively. In both of the flow is completely negligible or towards up- the pictures, smoke pieces are seen to move 


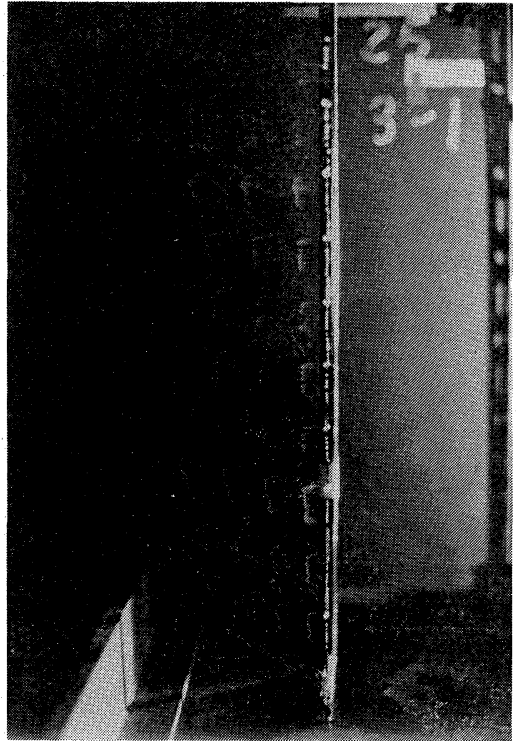

(a)

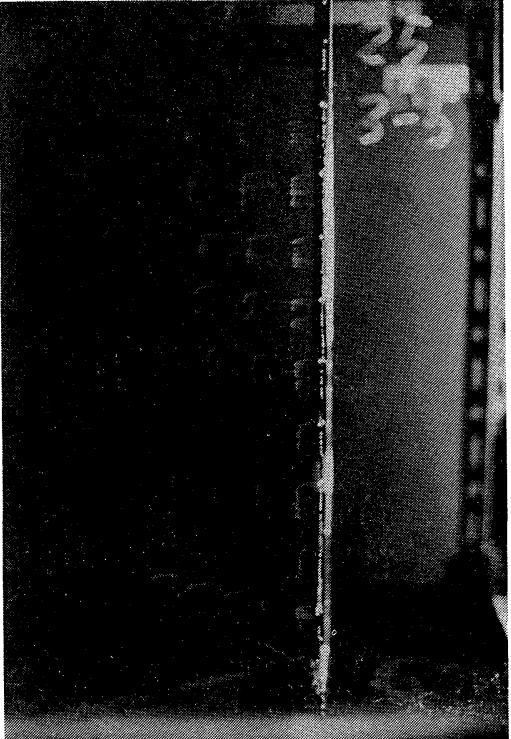

(b)

Fig. 4 Smoke pictures for stably stratified condition. $F_{r} \pi=0.71, b=0.08$. (a) $x / h=2.0$; (b) $x / h=4.0$.

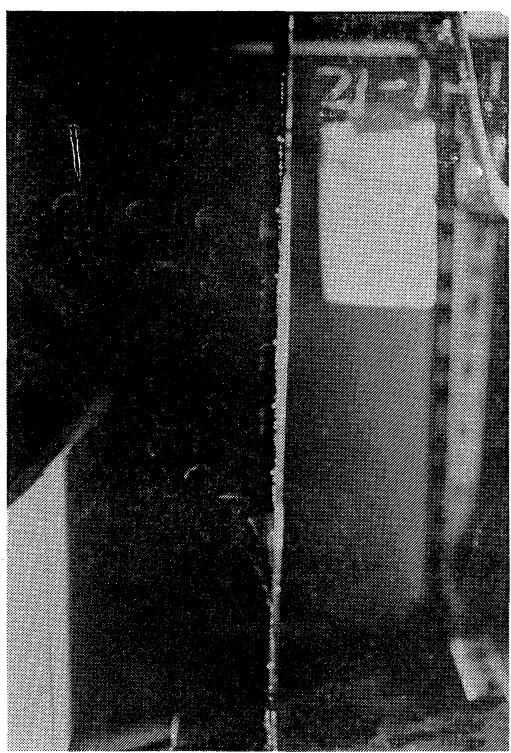

(a)

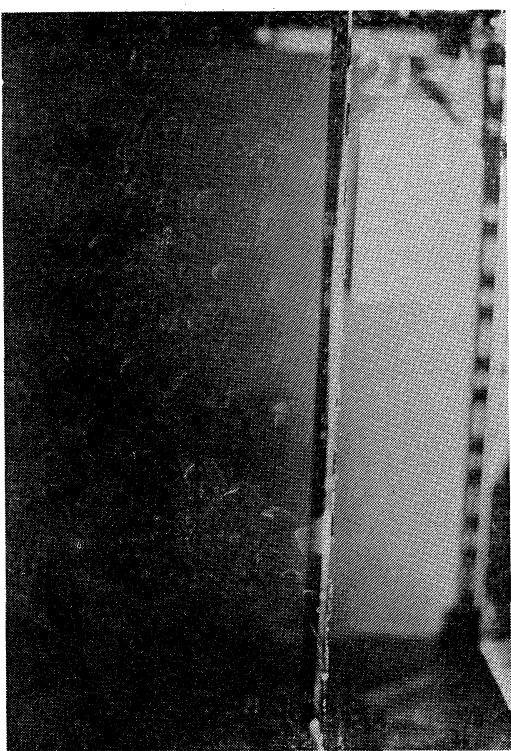

(b)

Fig. 5 Smoke pictures for stably stratified condition. $F r \pi=0.58, b=0.25$. (a) $x / h=0.67$; (b) $x / h=2.67$.

downstream and stagnant zone does not exist. the lower layer, we cannot recognize smoke The dependence of the flow stagnation on the pieces because of the formation of the stagnant obstacle height is thus suggested.

Figs. 5 and 6 show smoke pictures of the air- pieces in the lower layer do not move and overflow under the stronger stratification than Figs. lap with the support lod of the smoke wire and 3 and 4. Figs. 5(a) and (b) are for $F_{r \pi}=0.58$, are difficult to identify. It is seen that the stagnant $b=0.25$, at $x / h=0.67$ and 2.67 , respectively. In region is relatively thick even at $x / h=2.67$. 


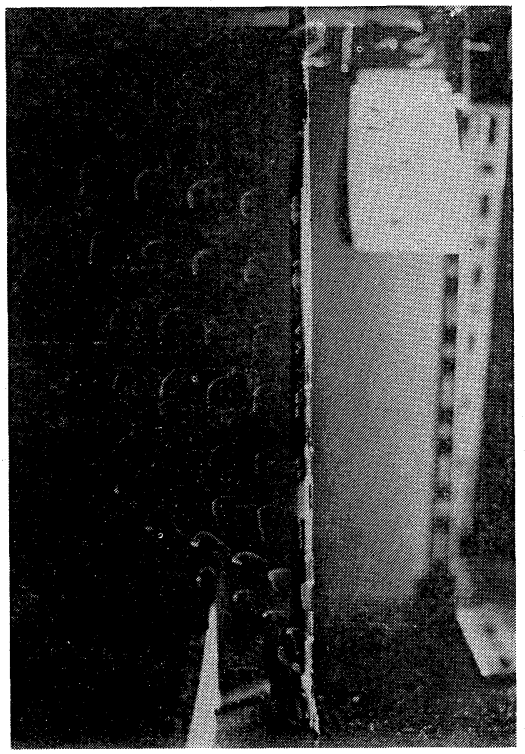

(a)

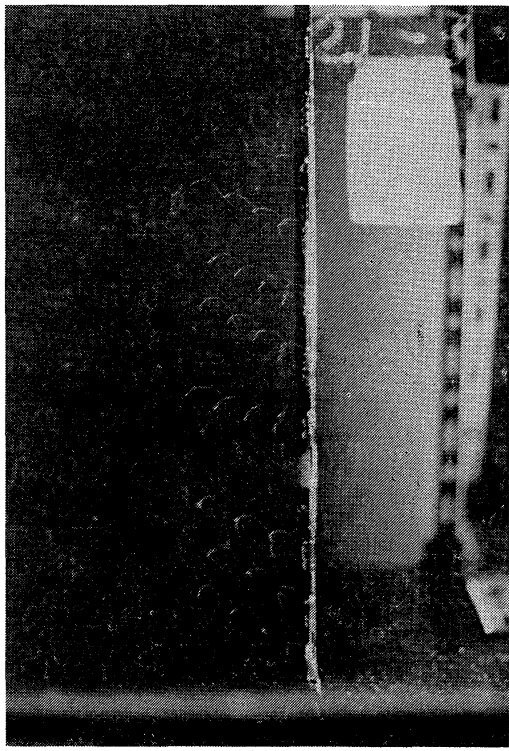

(b)

Fig. 6 Smoke pictures for stably stratified condition. $F r \pi=0.58, b=0.08$. (a) $x / h=1.0$; (b) $x / h=4.0$.

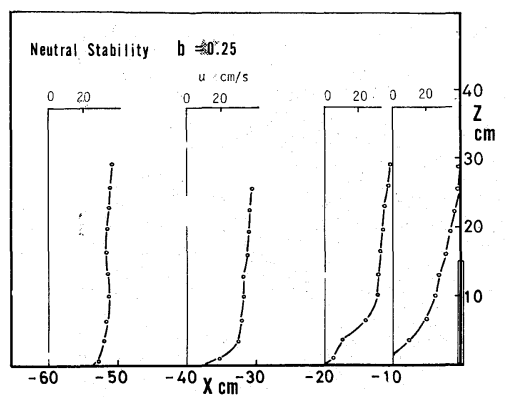

Fig. 7 Velocity profiles upstream of the barrier in the non-stratified flow, derived from the smoke wire pictures. $b=0.25$.

Smoke pictures under the similar stratification condition but for the barrier of $b=0.08$ are shown in Figs. 6(a) and (b). The former is at $x / h=1.0$ and the latter at 4.0. The stagnant zone was not recognized in this case.

From the smoke pictures, velocity profiles upstream of the barriers were derived. The flow velocities were calculated from the travel distances of the smoke pieces during the time duration of the strobo flash. Fig. 7 is the velocity profiles in the non-stratified flow upstream of the barrier of $b=0.25$. In the non-stratified flow, the stagnant region does not appear and the velocity profiles become almost constant with the height except the bottom layer, where the boundary layer developed affects the velocity profiles. In

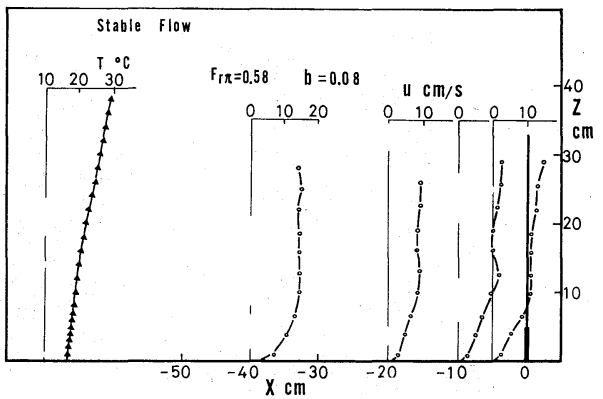

Fig. 8 Velocity profiles upstream of the barrier of $b=0.08$ in the stably stratified flow, Fr $\pi=0.58$.

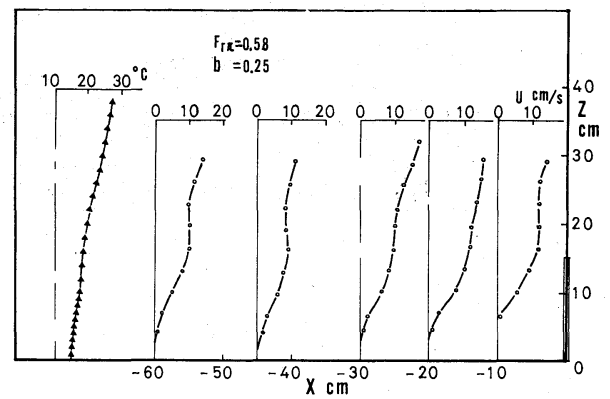

Fig. 9 Velocity profiles upstream of the barrier of $b=0.25$ in the stably stratified flow, $F_{r} \pi=0.58$.

the non-stratified flow, distortion of the velocity profiles due to the existing barrier were not detected at three or four times upstream of the 


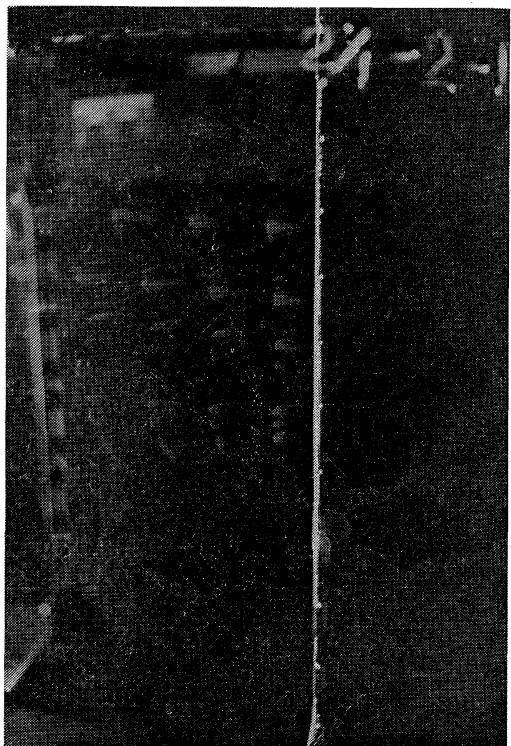

(a)

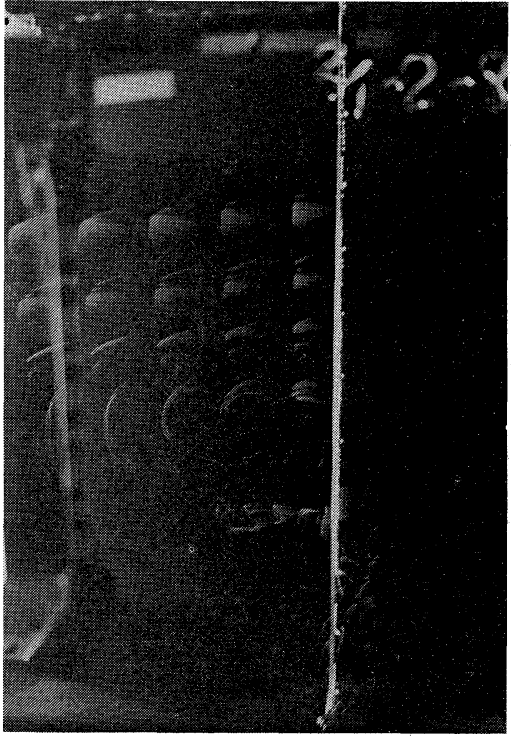

(b)

Fig. 10 Smoke pictures in the wake region of the barrier of $b=0.18$ under the non-stratified condition. The ambient flow is from right to left. (a) $x / h=1.8$; (b) $x / h=5.5$.

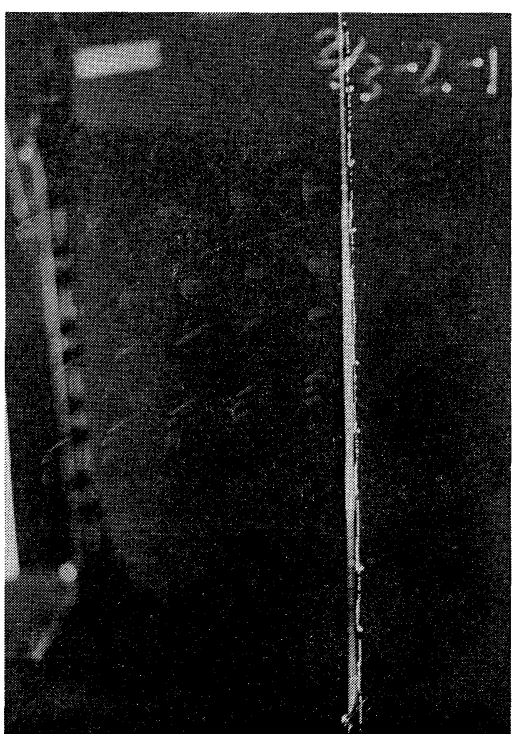

(a)

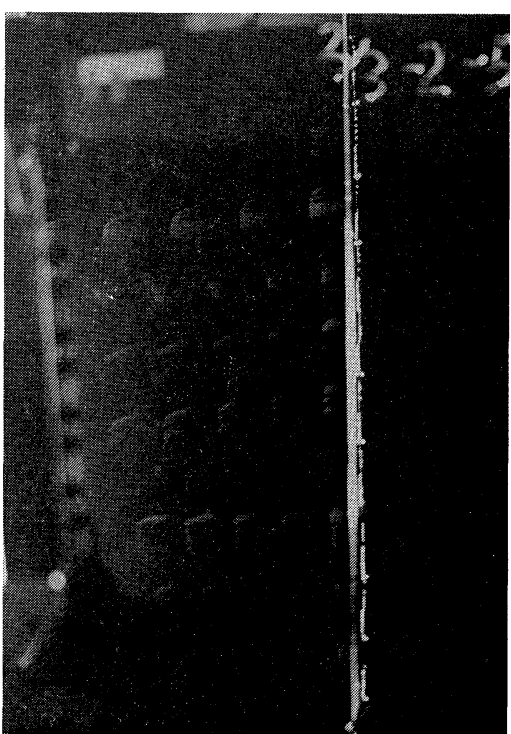

(b)

Fig. 11 Smoke pictures in the wake region of the stably stratified flow. $F r \pi=0.71, b=0.18$. (a) $x / h=1.8$; (b) $x / h=5.5$.

barrier height.

The velocity profiles in the stably stratified flows are shown in Figs. 8 and 9. These figures are for $F_{r \pi}$ equal to 0.58 and $b$ equal to 0.08 and 0.25 , respectively. The vertical profiles of airflow temperature at $60 \mathrm{~cm}$ upstream of the barrier are also shown. From the figures, it is seen that the flow stagnation exists at $F_{r \pi}$ equal to 0.58 for the barrier of $b=0.25$. The stagnant region extends far upstream of the barrier. For the barrier of $b=0.18$, the stagnant zone was observed at $F_{r \pi}=0.58$, but was not detected at $F_{r \pi}=0.71$. For the barrier of $b=0.08$, the stagnant region was neither found at $F_{r \pi}=0.58$ 


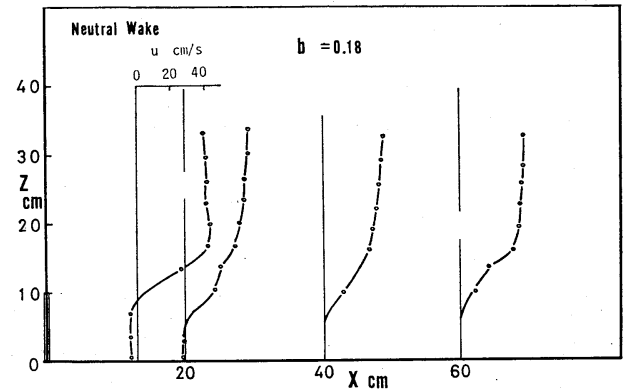

Fig. 12 Velocity profiles in the wake region of non-stratified flow, $b=0.18$.

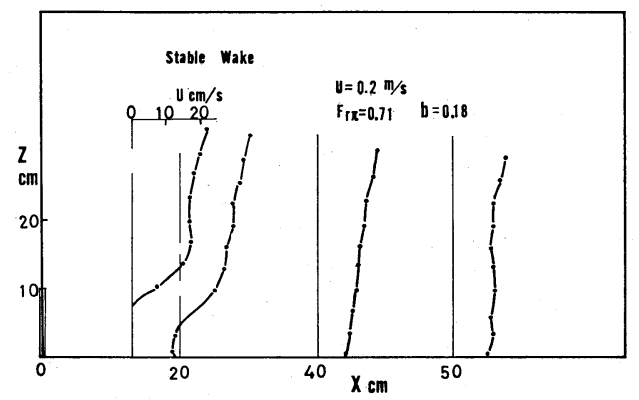

Fig. 13 Velocity profiles in the wake region of the stably stratified flow, $F r \pi=0.71, b=0.18$.

nor at 0.71 . Thus, the critical Froude number $F_{r \pi c}$ less than that a stagnant zone is formed is shown to vary with the barrier height.

\subsection{The airflow downstream of the barrier}

The velocity profiles downstream of the barriers have been also surveyed by the smoke wire system. The smoke pictures for the barrier of $b=0.18$ are displayed in Figs. 10 and 11 . Figs. 10(a) and (b) are pictures at $x / h=1.8$ and 5.5 in the non-stratified flow, respectively. Figs. 11(a) and (b) are the pictures at the same position with Fig. 10 but under the stably stratified condition of $F_{r \pi}=0.71$. From these figures, it is clearly seen that the wake region where the flow is reverse extends at least up to $x / h=5.5$ in the non-stratified flow, but it shrinks in the stably stratified flow.

The velocity profile downstream of the barrier in the non-stratified flow is shown in Fig. 12 for the barrier of $b=0.18$. In the non-stratified flow, the wake region is seen to extend further than five times of the barrier height. In the nonstratified flow, the streamwise scale of the wake region for the barrier of $b=0.08$ is about eight times of the barrier height. This wake scale is almost similar to the observed result by Ogawa
(1973) down-wind of a fence in the atmosphere.

Fig. 13 shows the velocity profiles in the wake under the stably stratified condition of $F_{r \pi}=0.71$ for $b=0.18$. The wake region is confined to the region four or five times downstream of the barrier height. The result suggests that the density stratification is effective to suppress the wake region, and is coincident with the recent theoretical and laboratory studies of wakes in density stratified flow by Brighton (1978) or Hunt et al. (1980).

\section{Theoretical consideration}

\subsection{The governing equation}

Stably stratified airflows in the atmosphere are reasonably assumed to be non-diffusive, nonviscous and incompressible for the scale of the airflow over a hill or a mountain. The effect of the rotation of the earth, i.e., Coriolis force is also negligible for the airflow of a scale less than tens of kilo-meters. Then, the airflow is possibly modelled by the wind tunnel flow discussed in the preceding section. The governing equations of density stratified flows in a channel are simplified to the following non-dimensional Helmholz type equation (see Yih (1958)):

$$
\nabla^{2} \Psi+F_{r_{\pi}}{ }^{-2} \Psi=-F_{r_{\pi}}{ }^{-2} \eta,
$$

where

$$
\begin{aligned}
& \nabla^{2}=\frac{\partial^{2}}{\partial \xi^{2}}+\frac{\partial^{2}}{\partial \eta^{2}}, \quad \Psi=\frac{\pi \Psi^{\prime}}{U H} \\
& \beta=\frac{H}{\rho_{0}}\left(\frac{\partial \rho}{\partial z}\right)_{x=-\infty}, \quad(\xi, \eta)=\frac{\pi}{H}(x, z), \\
& \frac{\partial \Psi^{\prime}}{\partial x}=w^{\prime}, \quad \frac{\partial \Psi^{\prime}}{\partial z}=-u^{\prime} .
\end{aligned}
$$

And $u^{\prime}$ and $w^{\prime}$ are the modified velocity components of the flow. According to Yih's expression, $u^{\prime}$ and $w^{\prime}$ are related to the velocity components $u$ and $w$ as

$$
\left(u^{\prime}, w^{\prime}\right)=\sqrt{\frac{\rho}{\rho_{0}}}(u, w),
$$

where $\rho$ is the density of the fluid and $\rho_{0}$ is the reference value of $\rho$.

\subsection{The method of solution}

The flow considered is a two-dimensional channel flow between two parallel plates separated by distance $H$ as shown in Fig. 14. A vertical plate of height $h$ is standing at $x=0$. The flow has a uniform incoming velocity and a linear density gradient at upstream infinity $(x=-\infty)$.

Here, we consider the flow where $F_{r \pi}$ is greater 


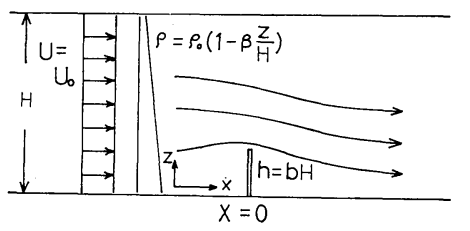

Fig. 14 Coordinate system of stably stratified flow over a barrier.

than unity. The solution is obtained for the upstream and downstream sides separately, as Oseen's model by Trustrum (1971). For the upstream side solution, the perturbation method by Hino and Onishi (1969) which extends the solution with respect to $1 / F_{r \pi}^{2}$ is applied.

\section{A. The upstream side solution}

The stream function upstream of the barrier is expanded as

$$
\Psi=-\eta+\Psi_{u}=-\eta+\phi_{0}+\delta \phi_{1}+\delta^{2} \phi_{2}+\cdots
$$

where $\delta$ is the inverse of the square of Froude number $F_{r \pi}$. Substituting (4-3) into (4-1) and arranging the term by order of $\delta$, we obtain following series of equations for $\phi_{i}$ :

$$
\begin{aligned}
& 0\left(\delta^{0}\right) ; \nabla^{2} \psi_{0}=0, \\
& 0\left(\delta^{\imath}\right) ; \nabla^{2} \psi_{i}=-\phi_{i-1} . \quad i \geq 1
\end{aligned}
$$

The solutions are obtained by solving (4-4) in increasing order of $i$, i.e. $\phi_{0}, \phi_{1}, \phi_{2}$ and so on.

The boundary conditions to be satisfied by $\Psi_{u}$ are

$$
\begin{aligned}
& \Psi_{u}=0, \quad \xi=-\infty \\
& \Psi_{u}=0, \quad \eta=\pi \\
& \Psi_{u}=0, \quad \xi \neq 0, \eta=0 \\
& \Psi_{u}-\eta=0, \quad 0 \leq \eta<b \pi, \xi=0 .
\end{aligned}
$$

Condition (4-5-a) is the so-called "Long's hypothesis" and it means that the disturbance produced by the barrier does not propagate to upstream infinity. Discussions have been given by McIntyre (1972), Baines (1977), Baines and Grimshow (1979) and others for this hypothesis. Baines and Grimshow suggested that the hypothesis is applicable when the obstacle is small and Froude number with respect to the obstacle height is sufficiently large. Wei et al. (1975) showed in a water channel experiment that the change of flow velocity upstream of a cylinder is less than 10 percents under moderate stratification condition. By these results, we can consider that the Long's hypothesis is sufficiently effective as long as stratification is moderate.

i) The solution $\phi_{0}$

As shown by (4-4-a), $\phi_{0}$ is the solution of the Laplace equation which satisfies the boundary conditions

$$
\begin{array}{ll}
\phi_{0}=0, & \xi=-\infty \\
\phi_{0}=0, & -\infty<\xi<0, \quad \eta=0 \\
\phi_{0}=0, & -\infty<\xi<0, \quad \eta=\pi \\
\phi_{0}=\eta, & \xi=0, \quad 0 \leq \eta<b \pi .
\end{array}
$$

The solution is given by

$$
\psi_{0}=\sum_{n=1}^{\infty} a_{n} e^{n \xi} \sin n \eta,
$$

where $a_{n}$ are constants which are determined by the conditions of $\phi_{0}$ at $\xi=0$. We introduce a function $g_{0}(\eta)$ which gives $\psi_{0}$ at $\xi=0$ as follows:

$$
\begin{aligned}
g_{0}(\eta) & =\eta, \quad 0 \leq \eta<b \pi, \\
& =\sum_{m} \alpha_{m} \frac{b \pi}{(\pi-b \pi)^{m}}(\pi-\eta)^{m}, \quad b \pi \leq \eta \leq \pi .
\end{aligned}
$$

The constants $\alpha_{m}$ are determined so that the function $\psi_{0}$ be a good approximation of the flow over a barrier under the non-stratified condition. Actually, flow induces a wake region in the lee of the barrier. But precise analytical model of the flow over a vertical barrier with a wake region in the lee is not available. Therefore, we introduced a flow over a semi-infinite step of height $b \pi$ and by which we approximated the flow over a barrier with a wake in the lee. The model flow was solved by a computer and the constants $\alpha_{m}$ were determined as the best approximation of the stream function profile at $\xi=0$. The obtained $\alpha_{m}$ are 0.5 for $m=2$ and 3, and otherwise zero.

The constants $a_{n}$ are given by the Fourier transformation of $g_{0}(\eta)$ as follows:

$$
\begin{aligned}
a_{n} & =\sum_{m} \alpha_{m}\left[\frac{2 \sin n \pi b}{n^{2}}-2 b(m !) \cos n \pi b\right. \\
& \times\left\{\frac{1}{(n \pi)^{3}(m-2) !(\pi-b \pi)^{2}}\right. \\
& +\frac{1}{(n \pi)^{5}(m-4) !(\pi-b \pi)^{4}}+2 b(m !) \\
& \times \sin n \pi b\left\{\frac{1}{(n \pi)^{2}(m-2) !(\pi-b \pi)^{2}}\right. \\
& \left.\left.+\frac{1}{(n \pi)^{4}(m-3) !(\pi-b \pi)^{3}}+\cdots\right\}\right] .
\end{aligned}
$$

ii) The higher order solutions

The solution of $\phi_{i}(i>1)$ can be solved with 
the solution of $\phi_{i-1}$. The boundary conditions to be satisfied by $\psi_{i}$ are

$$
\begin{array}{ll}
\phi_{i}=0, & \eta=0 \text { and } \eta=\pi, \\
\phi_{i}=0, & \xi=-\infty .
\end{array}
$$

The solution of $\phi_{i}$ for $i=1$ and 2 are given by

$$
\begin{aligned}
& \phi_{1}=\sum_{n=1}^{\infty}\left(A_{n}+B_{n} \xi\right) e^{n \xi} \sin n \eta, \\
& \psi_{2}=\sum_{n=1}^{\infty}\left(C_{n}+D_{n} \xi+E_{n} \xi^{2}\right) e^{n \xi} \sin n \eta
\end{aligned}
$$

where

$$
\begin{aligned}
& B_{n}=-\frac{a_{n}}{2 n} \\
& D_{n}=\frac{1}{n}\left(-\frac{A_{n}}{2}+\frac{B_{n}}{4 n}\right) \\
& E_{n}=\frac{a_{n}}{8 n^{3}} .
\end{aligned}
$$

As is readily clear from (4-11) and (4-12), the coefficients $A_{n}$ and $C_{n}$ are directly related to the distribution of $\phi_{1}$ and $\phi_{2}$ at $\xi=0$. In the present study, $A_{n}$ and $C_{n}$ are determined by applying Bernoulli's theorem to the flow. Details of the derivations are given in Appendix.

\section{B. The downstream side solution}

In the lee of an obstacle within the density stratified flow, well known lee waves occur and the flow properties are very different from the upstream. Here, we adopt the solution by Trustrum (1964), which was derived as limiting solution $t=\infty$ of an unsteady, density stratified flow over a thin vertical barrier. The solution is formally written by

$$
\begin{aligned}
\Psi_{d} & =-\eta+\sum_{n=1}^{k}\left(G_{n} \sin \lambda_{n} \xi+H_{n} \cos \lambda_{n} \xi\right) \sin n \eta \\
& +\sum_{n=k+1}^{\infty}\left(J_{n} e^{-} \lambda_{n} \xi+I_{n}\right) \sin n \eta,
\end{aligned}
$$

where $k$ is the maximum integer of $n$ which makes $\delta-n^{2}$ greater or equal to zero and $\lambda_{n}$ is

$$
\lambda_{n}=\left(\delta-n^{2}\right)^{1 / 2} .
$$

In the present model, the coefficients $G_{n}, H_{n}$, $I_{n}$ and $J_{n}$ are decided by the continuity conditions of $\Psi_{d}$ with the upstream solution $\Psi_{u}$ at $\xi=0$. The matching conditions are actually written by

$$
\begin{aligned}
& \Psi_{u, \xi=0-}=\Psi_{d, \xi=0^{+}} \\
& \left(\frac{\partial \Psi_{u}}{\partial \eta}\right)_{\xi=0^{-}}=\left(\frac{\partial \Psi_{d}}{\partial \eta}\right)_{\xi=0^{+}} \\
& \left(\frac{\partial \Psi_{u}}{\partial \xi}\right)_{\xi=0^{-}}=\left(\frac{\partial \Psi_{d}}{\partial \xi}\right)_{\xi=0^{+}}
\end{aligned}
$$

where the suffix $0^{-}$and $0^{+}$mean that $\xi$ is indefinitely close to zero in negative or positive space of $\xi$. The coefficients $G_{n}, H_{n}, I_{n}$ and $J_{n}$ cf the downstream solution are then written in terms of the coefficients of the upstream solution as follows:

$$
\begin{aligned}
& G_{n}=\frac{1}{\lambda_{n}}\left\{n a_{n}+\delta\left(n A_{n}-\frac{a_{n}}{2 n}\right)\right. \\
& \left.+\delta^{2}\left(n C_{n}-\frac{a_{n}}{8 n^{3}}-\frac{A_{n}}{2 n}\right)\right\} \\
& H_{n}=a_{n}+\delta A_{n}+\delta^{2} C_{n} \\
& I_{n}=a_{n}+\delta A_{n}+\delta^{2} C_{n}+\frac{1}{\lambda_{n}}\left\{n a_{n}+\delta\left(n A_{n}-\frac{a_{n}}{2 n}\right)\right. \\
& \left.+\delta^{2}\left(n C_{n}-\frac{a_{n}}{8 n^{3}}-\frac{A_{n}}{2 n}\right)\right\} \\
& J_{n}=-\frac{1}{\lambda_{n}}\left\{n a_{n}+\delta\left(n A_{n}-\frac{a_{n}}{2 n}\right)\right. \\
& \left.+\delta^{2}\left(n C_{n}-\frac{a_{n}}{8 n^{3}}-\frac{A_{n}}{2 n}\right)\right\} \text {. }
\end{aligned}
$$

\section{Discussions}

\subsection{The flow patterns by the theoretical model}

The streamlines for two barriers of different height and different stratification conditions are shown in Fig. 15 and 16. Froude number $F_{r \pi}$ are 3.3 and 0.71 . The theoretical model discussed in the chapter 4 cannot be applied to the flow of $F_{r \pi}$ less than unity in a strict sense. Therefore, Figs. 15(b) and 16(b) are only for reference. On the upstream side of the barrier, no apparent change of the flow appears for the small barrier of $b=0.1$. But for higher barriers for example $b=0.2$, the flow is suggested to change rapidly with $F_{r \pi}$ and in the range of small value of it, a vortex region is implied to be formed on the upstream side. The vorticity in this region is weak for the range of $F_{r \pi}$ near unity and we may consider this as a stagnant region. The largest $F_{r \pi}$ less than that a stagnant region is formed (we call this as the critical Froude number and express by $F_{r \pi c}$ ) is found to change together with the barrier height.

\subsection{The vertical profiles of flow velocity and density}

The wind tunnel results for velocity profiles upstream of the barrier are shown in Figs. 17 together with the theoretical results. Figs. 17(a) and (b) are the velocity profiles at $F_{r \pi}$ equal to 0.71 and 0.58 for the barrier of $b=0.25$. A stagnant region is clearly seen at $F_{r \pi}=0.71$. For the barrier of $b=0.18$, a stagnant region is not observed at $F_{r \pi}=0.71$. It is formed at $F_{r \pi}=0.58$. 


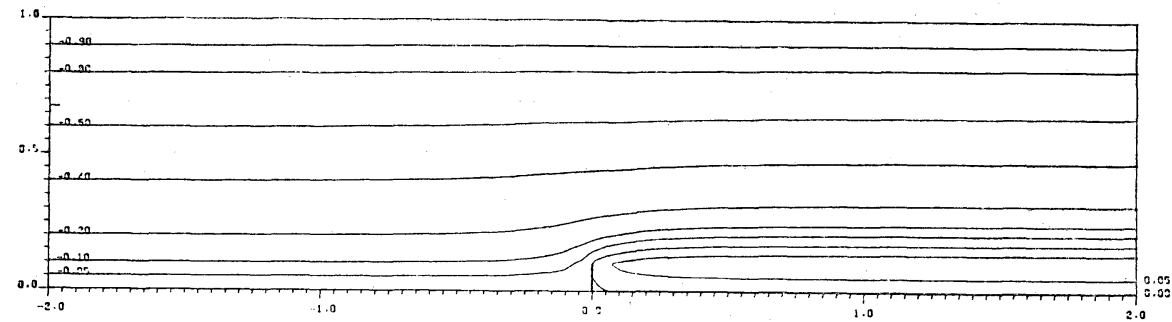

(a)

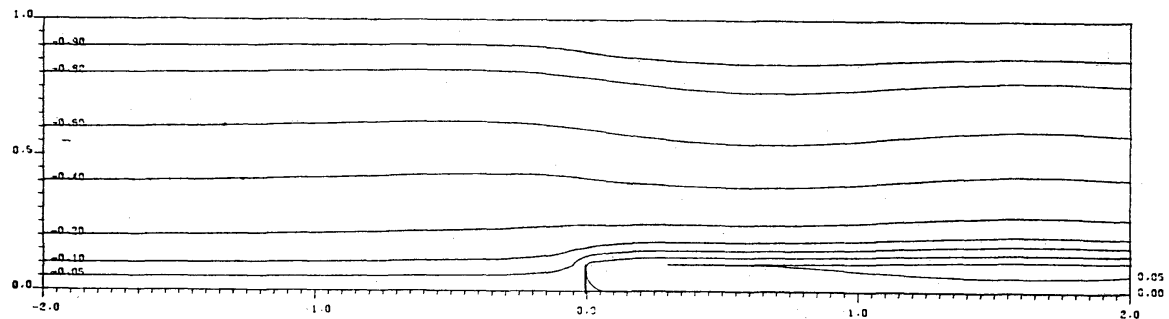

(b)

Fig. 15 Streamlines over a barrier by the theoretical model for $b=0.1$. (a) $F_{r \pi}=3.3$; (b) $F_{r \pi}=0.71$.

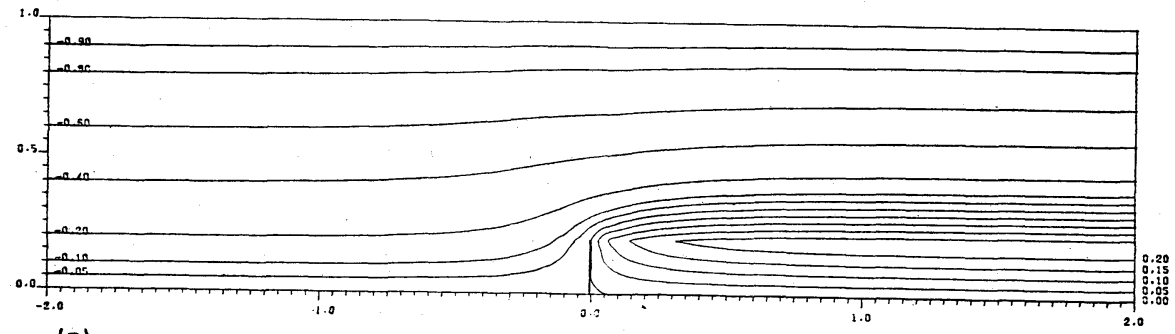

(a)

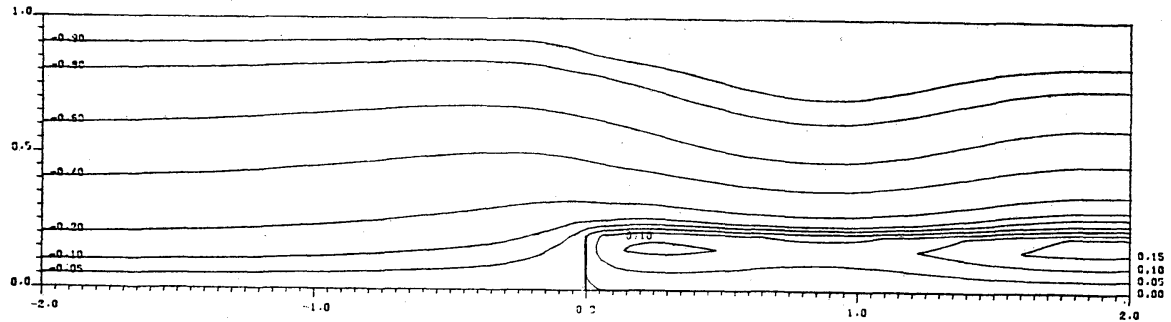

(b)

Fig. 16 Streamlines over the barrier of $b=0.20$ by the theoretical model. (a) $F_{r \pi}=3.3$;

(b) $F_{r \pi}=0.71$.

The velocity profiles by the theory agree satisfactorily with the experiment, except the lower layer near the floor. The disagreement may somewhat attribute to the viscosity, i.e. viscosity is neglected in the theory even it affects the velocitv profiles in the real flow.
The vertical profile of normalized fluid density by the experiment is shown in Fig. 18 together with the theoretical result. The deviation of the density from the reference is normalized by the density difference at the top and bottom of the fluid layer. The reference density is that of the 
(a)

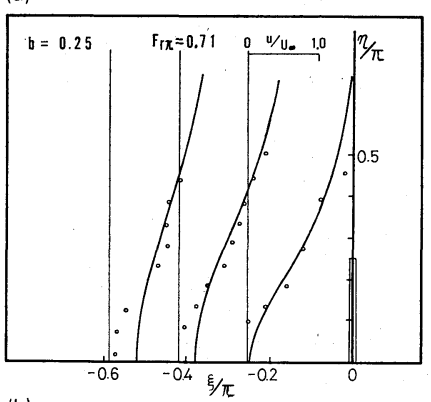

(b)

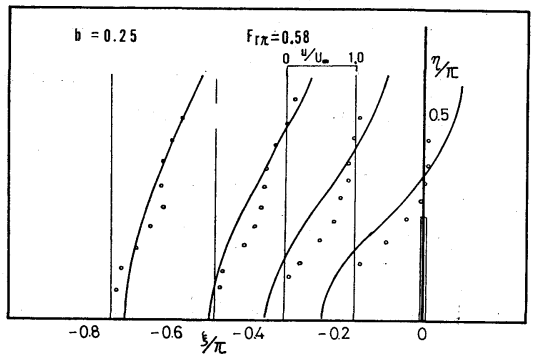

Fig. 17 Comparison of the velocity profiles upstream of the barrier of $b=0.25$ by the theoretical model and experiment. Open circles are by experiment. (a) $F r \pi=0.71$; (b) $F_{r} \pi=0.58$.

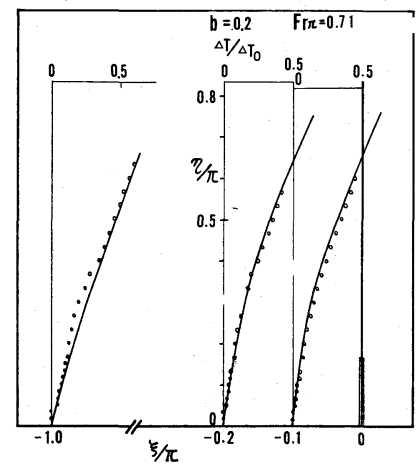

Fig. 18 Comparison of the density profiles upstream of the barrier by the theoretical model (solid line) and the experiment (open circles).

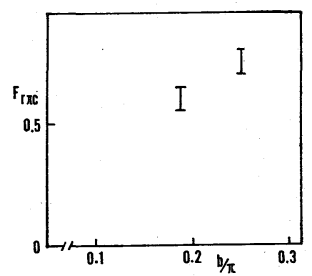

Fig. 19 The relation between the critical Froude number and the barrier height by the wind tunnel experiment. bottom layer. The density gradient in the lower layer is less than the upper layer and suggesting the flow blockage. The decrease of the density gradient in the stagnant region may show the effect of mixing even it is weak.

\subsection{The critical Froude number for the upstream stagnant region}

The estimated value of $F_{r \pi c}$ from wind tunnel experiment are shown in Fig. 19 with respect to the barrier height. As is easily seen, $F_{r \pi c}$ is nearly proportional to the barrier height. This means that if we adopt the barrier height as the length scale in Froude number, then, $F_{r \pi c}$ becomes constant regardless of the barrier height. The critical Froude number in terms of the barrier height (denoted by $F_{r \pi b c}$ ) is given by

$$
F_{r \pi b c}=\frac{U_{c} \pi}{N b H} .
$$

$F_{r \pi b c}$ is almost independent to the barrier scale. The theoretical model suggests that a stagnant region is formed at larger Froude number for larger barrier.

\section{Concluding remarks}

For flow blocking upstream of an obstacle, Long (1953) first suggested and Kao (1965) later discussed an analytical model. Kao took the Froude number with respect to the channel height as the controlling parameter of the flow and discussed the change of the flow due to the Froude number. But as suggested by Sheppard (1956) and recently by Baines and Grimshow (1979), the flow blockage may not be independent to the obstacle height and the Froude number with respect to the obstacle height may be a suitable parameter of the flow. The relation between the critical Fro le number and the obstacle height is the primary concern of this study.

The formation and the characteristics of flow blockage were studied by a wind tunnel experiment and also by a theoretical model. The obstacle was simplified to a thin vertical barrier to exclude the effect of the slope angle to the flow. The wind tunnel observation of the airflow by a smoke wire clearly showed that a stagnant region is formed upstream of the barrier when the Froude number is sufficiently small. The critical Froude number by the experiment is shown to be proportional to the barrier height. And it is also supported by the theoretical model. For the barrier of non-dimensional height $b=$ 0.25 which is equal to the obstacle height in Kao's study, the critical value $F_{r \pi c}$ is nearly equivalent 
to the value suggested by Long. A more adequate parameter of the flow is the Froude number with respect to the barrier height. According to this Froude number, the critical value is about $\pi$ and becomes independent to the barrier height.

The wake region downstream of the barrier is also discussed experimentally. The downstream wake is shown to decay as upstream stagnation occurs. The wake region is observed to shrink together with increase of the stagnation effect.

\section{Acknowledgements}

The author wishes to express his sincere thanks to Professor M. Hino of Tokyo Technical College for his helpful advice and encouragement through the study. $\mathrm{He}$ also wishes to express his thanks to Dr. O. Yokoyama and another staff members of National Research Institute for Pollution and Resources for their helpful discussions and cooperation.

\section{Appendix: The coefficients $A_{n}$ and $C_{n}$}

It is known that

$$
H_{e}=\frac{P}{\rho_{0}}+\frac{1}{2} U_{\infty}^{2}\left(u^{2}+w^{2}\right)+\frac{\rho g H}{\rho_{0} \pi} \eta
$$

is conserved along a streamline in our system, i.e., Bernoulli's constant $H_{e}$ is a function of $\psi$ only, where $P$ is the pressure. Let us calculate the coefficients $A_{n}$ and $C_{n}$.

1. $H_{e}$ at $\xi=0$

We will derive the pressure, inertia and potential terms of Bernoulli's constant separately.

(1-a) The pressure term at $\xi=0$

The static pressure at $\xi=0$ is expressed by

$$
\frac{P}{\rho_{0}}=\frac{P_{\infty}}{\rho_{0}}+\frac{g H}{\rho_{0} \pi} \int_{\eta}^{\pi} \rho d \eta+f(U),
$$

where the first term on the right-hand side is the pressure at upstream infinity and upper boundary, i.e., $\xi=-\infty$ and $\eta=\pi$. The second term is the perturbation of the pressure due to the vertical deviation of the streamline from its original height (i.e., at upstream infinity). The third term is the change of the cross sectional average pressure due to the decrease of the flow cross section by the barrier. Since the flow is non-diffusive and the density of the fluid is only a function of the stream function, the second term is given by

$$
\begin{aligned}
& \frac{g H}{\rho_{0} \pi} \int_{\eta}^{\pi} \rho d \eta=g \frac{H}{\pi}(\pi-\eta)-\frac{1}{2} \frac{g H \beta}{\pi^{2}}\left(\pi^{2}-\eta^{2}\right) \\
& \quad-\frac{g H \beta}{\pi^{2}} \sum_{n=1}^{\infty} \frac{1}{n}\left[\left(a_{n}+\delta A_{n}+\delta^{2} C_{n}\right)\right. \\
& \left.\quad \times\left\{(-1)^{n}-\cos n \eta\right\}\right] .
\end{aligned}
$$

For the first order approximation, the third term $f(U)$ is considered to be independent of height, and reasonably be assumed to be equal to the average excess of the flow velocity from upstream infinity, i.e.,

$$
\overline{f(U)=-\frac{1}{2} U_{\infty}{ }^{2}\left[\left\{-1+\sum_{n=1}^{\infty} n a_{n} \cos n \eta\right\}^{2}-1\right]},
$$

where overbar denotes the average with respect to $\eta(\eta=b \pi$ to $\pi)$.

(1-b) The inertia term at $\xi=0$

In the inertia term, the vertical component of velocity is small compared to the horizontal and negligible. Then the inertia force $q^{2 / 2}$ is given by

$$
\begin{aligned}
\frac{1}{2} q^{2} & =\frac{1}{2} U_{\infty}{ }_{\infty}\left\{-1+\sum_{n=1}^{\infty} n a_{n} \cos n \eta\right\}^{2} \\
+ & 2\left\{-1+\sum_{n=1}^{\infty} n a_{n} \cos n \eta\right\} \\
& \times\left(\sum_{n=1}^{\infty}\left(\delta n A_{n}+\delta^{2} n C_{n}\right) \cos n \eta\right) \\
& +\left(\sum_{n=1}^{\infty}\left(\delta n A_{n}+\delta^{2} n C_{n}\right) \cos n \eta\right)^{2}
\end{aligned}
$$

(1-c) The potential term

This term is given by rewriting $\rho$ in the last term in (A-1) by

$$
\rho=\rho_{0}\left(1+\frac{\beta}{\pi} \Psi\right)
$$

i.e.,

$$
\frac{\rho g H}{\rho_{0} \pi}=\frac{g H}{\pi} \eta\left(1+\frac{\beta}{\pi} \Psi\right) .
$$

By the eqs. (A-3), (A-4), (A-5) and (A-7), all terms in Bernoulli's sum $H_{e}$ at $\xi=0$ are determined.

2. Bernoulli's constant at $\xi=-\infty$

(2-a) The pressure term

When the vertical position of the streamline which passes through $\eta$ at $\xi=0$ is given by $\eta^{\prime}$ at $\xi=-\infty$, the static pressure at $\xi=-\infty$ is expressed as follows:

$$
\frac{P}{\rho_{0}}=\frac{P_{\infty}}{\rho_{0}}+g \frac{H}{\pi} \int_{\eta^{\prime}}^{\pi}\left(1-\beta \eta^{\prime \prime}\right) d \eta^{\prime \prime} .
$$

Since $\eta^{\prime}$ is equal to $-\Psi$ at $\xi=-\infty,(\mathrm{A}-8)$ is rewritten as follows:

$$
\begin{aligned}
\frac{P}{\rho_{0}} & =\frac{P_{\infty}}{\rho_{0}}+\frac{g H}{\pi} \sum_{n=1}^{\infty}\left(a_{n}+\delta A_{n}+\delta^{2} C_{n}\right) \sin n \eta \\
& +\frac{g H}{\pi}(\pi-\eta)-\frac{1}{2} \frac{g H \beta}{\pi^{2}}\left(\pi^{2}-\eta^{2}\right)
\end{aligned}
$$




$$
\begin{aligned}
& -\frac{g H \beta}{\pi^{2}} \eta \sum_{n=1}^{\infty}\left(a_{n}+\delta A_{n}+\delta^{2} C_{n}\right) \sin n \eta \\
& +\frac{1}{2} \frac{g H \beta}{\pi^{2}}\left(\sum_{n=1}^{\infty}\left(a_{n}+\delta A_{n}+\delta^{2} C_{n}\right) \sin n \eta\right)^{2} .
\end{aligned}
$$

(2-b) The inertia term

At $\xi=-\infty$, the flow velocity is uniform regardless of height and equal to. $U_{\infty}$, therefore, inertia term is simply given by

$$
\frac{1}{2} q^{2}=\frac{1}{2} U_{\infty}{ }^{2} \text {. }
$$

(2-c) The potential term

As in (2-a), a streamline which passes through $\eta$ at $\xi=0$ is assumed to pass through $\eta^{\prime}$ at $\xi=$ $-\infty$. Then, from the equality of $\eta$ and $-\Psi$ at $\xi=-\infty$, the potential term is given by

$$
\begin{gathered}
\frac{\rho g H \eta^{\prime}}{\rho_{0} \pi}=\frac{g H}{\pi} \cdot\left(\eta-\sum_{n=1}^{\infty}\left(a_{n}+\delta A_{n}\right.\right. \\
\left.\left.+\delta^{2} C_{n}\right) \sin n \eta\right)\left(1+\frac{\beta}{\pi} \Psi\right) .
\end{gathered}
$$

3. The equation of $A_{n}$ and $C_{n}$

Equating Bernoulli's constants at $\xi=0$ and $\xi=-\infty$ and arranging resultant equation by the order of $\delta$, we obtain the equations from which $A_{n}$ and $C_{n}$ are determined as follows:

$$
\begin{aligned}
0\left(\delta^{1}\right) ; F \sum_{n=1}^{\infty} n A_{n} \cos n \eta=\frac{1}{2}\left(\sum_{n=1}^{\infty} a_{n} \sin n \eta\right)^{2} \\
\quad-\sum_{n=1}^{\infty} \frac{a_{n}}{n}\left\{(-1)^{n}-\cos n \eta\right\}, \quad(\mathrm{A}-12) \\
0\left(\delta^{2}\right) ; F \sum_{n=1}^{\infty} n C_{n} \cos n \eta \\
=\sum_{n=1}^{\infty} \frac{A_{n}}{n}\left\{(-1)^{n}-\cos n \eta\right\} \\
\quad+\frac{1}{2}\left(\sum_{n=1}^{\infty} a_{n} \sin n \eta\right)\left(\sum_{n=1}^{\infty} A_{n} \sin n \eta\right) \\
-\frac{1}{2}\left(\sum_{n=1}^{\infty} n A_{n} \cos n \eta\right)^{2}
\end{aligned}
$$

where $F$ is given by

$$
F=2\left(-1+\sum_{n=1}^{\infty} n a_{n} \cos n \eta\right) \text {. }
$$

For the first approximation $F$ is considered to be a constant.

From (A-12) and (A-13) coefficients $A_{n}$ and $C_{n}$ are determined by the Fourier transformation.

\section{References}

Baines, P. G., 1977: Upstream influence and Long's model in stratified flows, Jour. Fluid Mech., 82, 147-159.

and Grimshow, R. H. J., 1979: Stratified flow over finite obstacle with weak stratification, Geophy. Astrophy. Fluid Dynamics, 13, 317-334.

Brighton, P.W. M., 1978: Strongly stratified flow past three-dimensional obstacles, Quart. Jour. Roy. Met. Soc., 104, 289-307.

Förchtgott, J., 1949: Wave streaming in the lee of mountain ridge, Bull. Met. Tchecosl., Prague, 3.

Hino, M. and Onishi, S., 1969: Effect of a pointsink location on flow regime of stratified fluid, Jour. of the Japan Soc. of Civil Eng., 163, 3948. (in Japanese)

, 1970: Analysis of uniformly stratified flow into a circular slot, Jour. of the Japan Soc. of Civil Eng., 178, 37-46. (in Japanese)

Hunt, J. R.C. and Snyder, W. H., 1980: Experiments on stably and neutrally stratified flow over a model three-dimensional hill, Jour. Fluid Mech., 96, 671-704.

Kao, T. W., 1965: The phenomenon of blocking in stratified fluid, Jour. of Geophy. Res., 70, 815822.

Kitabayashi, K., 1977: Wind tunnel and field studies of stagnant flow upstream of a ridge, Jour. Met. Soc. of Japan, 55, 193-204.

Long, R. R., 1953: Some aspect of the flow of stratified fluids I, A theoretical investigation, Tellus, 5, 42-57.

McIntyre, M. E.. 1972: On Long's hypothesis of no upstream influence in uniformly stratified or rotating fluid, Jour. of Fluid Mech., 52, 209-243.

Ogawa, Y., 1973: Effects of building and thermal boundary layer on diffusion, Ph. D. Thesis, Hokkaido University, Japan.

Sheppard, P. A., 1956: Airflow over mountains, Quart. Jour. of Roy. Met. Soc., 82, 528-529.

Trustrum, K., 1964: Rotating and stratified fluid flow, Jour. of Fluid Mech., 19, 415-432.

- 1971: An O'seen Model of the twodimensional flow of a stratified flow over an obstacle, Jour. of Fluid Mech., 50, 177-188.

Wei, S. N., Kao, T. W. and Pao, Y.H., 1975: Experimental study of upstream influence in the two-dimensional flow of a stratified fluid over an obstacle, Geophy. Fluid Dynamics, 6, 315-336. Yih, C. S., 1958: On the flow of a stratified fluid, Proc. 3 rd U.S. National Congress of Appl. Mech. 


\title{
密度成層気流中におかれた障壁上流側のよどみ域について
}

\author{
北 林 興 ニ \\ 公害資源研究所
}

大気が安定成層状態にある時, 山岳あるいは構築物の上流側に “よどみ域”が発生する。このよどみ域の発生 条件を風洞実験および，理論モデルにより検討した。

山岳あるいは構築物を二次元薄板障壁でモデル化し, 温度成層をなす風洞中で流れの観察を行った。その結果, フルード数がある臨界值より小さい場合に，障壁上流側に気塊の動きの少ない，よどみ域が形成されることが確 められた。

また，流れを非粘性，密度成層流とし，Perturbation 法で解くことによりフコルド数による流れの変化を 検討した。風洞実験の結果, よどみ域の発生する臨界フルード数 $F_{r \pi c}=U_{c} / N H$ は障壁高さにほぼ比例すること が明らかとなり，この結果は理論モデルの結果とも整合する。 